\title{
A Question of Digital Linear Algebra
}

\author{
Yan Gérard \\ Laboratoire de Logique et d'Informatique de Clermont1 (LLAIC1) IUT \\ Département d'Informatique, Ensemble Universitaire des Cézeaux \\ B.P. 86, 63172 Aubière Cedex, France \\ gerard@llaic.u-clermont1.fr
}

\begin{abstract}
In classical linear algebra, the question to know if a vector $v \in \mathbb{R}^{n}$ belongs to the linear space $V e c t\left\{v^{1}, v^{2}, \cdots, v^{k}\right\}$ generated by a familly of vectors, is solved by the Gauss pivot. The problem investigated in this paper is very close to this classical question: we denote $\lfloor.\rfloor_{n}$ the function of $\mathbb{R}^{n}$ defined by $\left\lfloor\left(x_{i}\right)_{1 \leq i \leq n}\right\rfloor_{n}=\left(\left\lfloor x_{i}\right\rfloor\right)_{1 \leq i \leq n}$ and the question is now to determine if a given vector $v \in \mathbb{Z}^{n}$ belongs to $\left\lfloor V e c t\left\{v^{1}, v^{2}, \cdots, v^{k}\right\}\right\rfloor_{n}$. This problem can be easily seen as a sytem of inequalities and solved by using linear programming but in some special cases, it can also be seen as a particular geometrical problem and solved by using tools of convex geometry. We will see in this framework that the question $v \in\left\lfloor V \operatorname{ect}\left\{v^{1}, v^{2}, \cdots, v^{k}\right\}\right\rfloor_{n}$ ? generalizes the problem of recognition of the finite parts of digital hyperplanes and we will give equivalent formulations which allow to solve it efficiently.
\end{abstract}

Keywords: chord, convex hull, digital hyperplane, simplex, slice.

\section{Introduction}

The problem that we are going to consider in this paper belongs to a set of questions of geometry of numbers, which have been deeply investigated since the beginning of the century. The problem which can be considered as the origin of the others, is the one which consists in computing if a given vector $v \in \mathbb{R}^{n}$ belongs to the linear subspace generated by a familly $v^{1}, v^{2}, \ldots . v^{k}$ of $\mathbb{R}^{n}$ :

$$
v \in V \operatorname{ect}\left\{v_{i} / 1 \leq i \leq k\right\} ?
$$

The method of computation used for solving this question is the very classical GAuss pivot. Many interessant problems are close to this easy and basic question: if we consider no more the linear space $\operatorname{Vect}\left\{v_{i} / 1 \leq i \leq k\right\}$ but only the additive group $\operatorname{Group}\left\{v_{i} / 1 \leq i \leq k\right\}=\left\{\sum_{i=1}^{k} a_{i} v^{i} / \forall 1 \leq i \leq k, a_{i} \in \mathbb{Z}\right\}$ generated by the vectors $v^{i}$, the problem is a system of diophantine equations. We could also be interested in the distance between $v$ and $V \operatorname{ect}\left\{v_{i} / 1 \leq i \leq k\right\}$ or $v$ and $\operatorname{Group}\left\{v_{i} / 1 \leq i \leq k\right\}$ instead of the single membership of $v$ to these sets. If the choosen distance comes from a defined quadratic form, it leads to classical computations but it is not the case if one makes the choice of the distance $d_{1}$ 
associated with the norm $N_{1}\left(x_{i}\right)_{1 \leq i \leq n}=\sum_{i=1}^{n}\left|x_{i}\right|$ or of the distance $d_{\infty}$ associated with the classical norm $N_{\infty}\left(x_{i}\right)_{1<i<n}=\max \left\{\left|x_{i}\right| / 1 \leq i \leq n\right\}$. We can mention KRONECKER's approximation theorem which provides a characterization of the vectors $v$ whose distance $d_{\infty}\left(v, \operatorname{Group}\left\{v_{i} / 1 \leq i \leq k\right\}\right)$ to the group Group $\left\{v_{i} / 1 \leq i \leq k\right\}$ is stricly less than a given $\epsilon$ [S 88$]$.

The question investigated in this paper is of the same kind: we introduce the function $\lfloor\cdot\rfloor_{n}: \mathbb{R}^{n} \longrightarrow \mathbb{Z}^{n}$ defined by $\left\lfloor\left(x_{i}\right)_{1 \leq i \leq n}\right\rfloor_{n}=\left(\left\lfloor x_{i}\right\rfloor\right)_{1 \leq i \leq n}$ where $\lfloor r\rfloor$ denotes the integer part of a real number $r$ (in other words, the largest integer $i$ verifying $i \leq r$ ) and the question is to determine whether a vector $v \in \mathbb{Z}^{n}$ belongs to the image of $\operatorname{Vect}\left(v^{1}, v^{2}, \ldots, v^{k}\right)$ by the map $\lfloor\cdot\rfloor_{n}$ :

$$
v \in\left\lfloor\operatorname{Vect}\left(v^{1}, v^{2}, \ldots, v^{k}\right)\right\rfloor_{n} ?
$$

The problem of determining if $v \in\left\lfloor V \operatorname{Vect}\left(v^{1}, v^{2}, \ldots, v^{k}\right)\right\rfloor_{n}$ does not appear in the literature of geometry of numbers and we can give two reasons to explain this absence:

- the first one is that the function integer part has never been considered as a serious source of problems in classical mathematics and if it is now the case, it is especially a preoccupation of computer scientists for which the truncature is a usual practical tool.

- the second reason comes from the fact that the analytical form of the question is quite ordinary. It is simply a system of inequalities: by denoting $v_{j}$ the coordinates of $v \in \mathbb{Z}^{n}$ and $v_{j}^{i}$ the coordinates of $v^{i} \in \mathbb{R}^{n}$, the problem is to determine whether there exist $k$ real numbers $a_{1}, a_{2}, \ldots, a_{k}$ verifying the system of $n$ double inequalities

$$
v_{j} \leq \sum_{i=1}^{k} a_{i} v_{j}^{i}<v_{j}+1
$$

for $1 j \leq n$. The systems of linear inequalities is of the domain of linear programming and there exists nowadays many different methods to solve them. The famoust one is the simplex method [Ga60] and we will come back later on its geometrical meaning in order to provide geometrical efficient algorithms. A second solution is the classical method of FOURIER-MOTZKIN which consists in eliminating the variables. A third one is an algorithmic technique eliminating the redundant linear inequalities and described in [E87]. There exists many others but the aim of this paper is not to present them. Our ambition is mainly to bring out the geometry which can be hidden in our special kind of systems. It is the reason why we are going to choose a geometrical point of view. We are however unable to do it in the general case and then we have to restict ourself to two special cases:

- when the rank of the familly $v^{1}, v^{2}, \ldots . v^{k}$ is $n$ or $n-1$, technics of linear algebra allow to conclude,

- when the constant vector $(1)_{1 \leq i \leq n}$ belongs to the linear subspace 
$V e c t\left(v^{1}, v^{2}, \ldots, v^{k}\right)$, the question to know whether $v \in\left\lfloor V e c t\left(v^{1}, v^{2}, \ldots, v^{k}\right)\right\rfloor_{n}$ generalizes a problem which has been discussed many times in the previous DGCI and which consists in recognizing the finite parts contained by naive digital hyperplanes ([DR94,D95,FST96,R95]). We will give a geometrical characterization of the solutions by introducing a new tool, the chords' set of a part of $\mathbb{R}^{n}$, and a classical notion, the convex hull. This geometrical characterization provides two natural algorithms and as the question generalizes the recognition of the digital hyperplanes, they can naturally be used to determine if a finite part belongs to a digital hyperplane of double inequality $h \leq \sum_{i=1}^{n} a_{i} x_{i}<h+\left|a_{n}\right|$.

As conclusion of this introduction, we have to say a word about the applications and we can begin with the recognition of the digital hyperplanes. A good algorithm of recognition can be the origin of many applications in digital imagery and it can lead to a whole set of geometrical methods of treatment of images. We have however to stress the applications of the main problem that we have considered because it has an inexhaustible number of numeric applications: one of them is the use of what we could call the polynomial approximations of the digital functions. We call polynomial approximation of a digital function $f: \mathbb{Z}^{d} \longrightarrow \mathbb{Z}$ under a domain $D \subset \mathbb{Z}^{d}$ a polynomial $P\left(X_{i}\right)_{1 \leq i \leq n}$ verifying for all $x \in D$,

$$
f(x)=\lfloor P(x)\rfloor .
$$

These objects provides values $\left(d^{k} P / d x_{i_{1}} . d x_{i_{2}} \ldots . . d x_{i_{k}}\right)$ which can be seen as the differentials of the function $f$. The computation of a polynomial approximation of degree less than $r$ for a function $f$ under a domain $D$ supposed ordered and of cardinality $n$ consists in determining a linear combination of the vectors $(1)_{i \in D},(i)_{i \in D},\left(i^{2}\right)_{i \in D}, \ldots .,\left(i^{r}\right)_{i \in D}$ whose image by $\lfloor\cdot\rfloor_{n}$ is $(f(i))_{i \in D}$. Then it is a direct application of the second case that we have planned to investigate. We can also approach the function $f$ by other things as polynomials and use it to create digital filters or many other things that we have not place enough to describe.

\section{Easy Cases}

The general question in which we are interested, consists in determining if a given vector $v \in \mathbb{Z}^{n}$ belongs to the image of the linear subspace

$V \operatorname{ect}\left\{v^{i} / 1 \leq i \leq k\right\}$ by the map $\lfloor\cdot\rfloor_{n}$.

The first easy case is trivial: when the rank of the familly $v^{1}, v^{2}, \ldots, v^{k}$ is $n$, the linear subspace $V \operatorname{ect}\left\{v^{i} / 1 \leq i \leq k\right\}$ is equal to the whole space $\mathbb{R}^{n}$ and as $\lfloor\cdot\rfloor_{n}$ is sujective, it is clear that the intersection of the reciprocal image of any $v \in \mathbb{Z}^{n}$ by $\lfloor\cdot\rfloor_{n}$ with $V \operatorname{ect}\left\{v^{i} / 1 \leq i \leq k\right\}$ can not be empty.

The second easy case is not trivial but it is not necesary to use the double inequalities to understand what happens : when the rank of the familly $v^{1}, v^{2}, \ldots, v^{k}$ is $n-1$, the linear subspace $V \operatorname{ect}\left\{v^{i} / 1 \leq i \leq k\right\}$ generated by these elements can be characterized by a linear form $\varphi$ (then we have $V \operatorname{ect}\left\{v^{i} / 1 \leq i \leq k\right\}=\{x \in$ $\left.\left.\mathbb{R}^{n} / \varphi(x)=0\right\}\right)$. We denote $a$ a point of the set $\{0,1\}^{n}$ where $\varphi$ is minimum 
and $b$ a point of $\{0,1\}^{n}$ where $\varphi$ is maximum. By denoting $\bigsqcup_{n}^{-1}(v)$ the reciprocal image of $v$ by $\lfloor\cdot\rfloor_{n}$, we can see that

$$
] \varphi(v+a) ; \varphi(v+b)\left[\subset \varphi\left(\lfloor\rfloor_{n}^{-1}(v)\right) \subset[\varphi(v+a) ; \varphi(v+b)] .\right.
$$

There are two trivial consequences:

- if 0 belongs to $] \varphi(v+a) ; \varphi(v+b)\left[\right.$, then $v$ belongs to $\left\lfloor V e c t\left\{v^{i} / 1 \leq i \leq k\right\}\right\rfloor_{n}$.

- if 0 does not belong to $[\varphi(v+a) ; \varphi(v+b)]$, then $v$ does not belong to the set $\left\lfloor V e c t\left\{v^{i} / 1 \leq i \leq k\right\}\right\rfloor_{n}$.

and there remains only one case: the one where one of the two values $\varphi(v+a)$ or $\varphi(v+b)$ is null. In that case, we just have to compute $\varphi(v)$ :

- if $\varphi(v)=0$ then $v$ belongs to $V e c t\left\{v^{i} / 1 \leq i \leq k\right\}$ and then also to its image $\left\lfloor V e c t\left\{v^{i} / 1 \leq i \leq k\right\}\right\rfloor_{n}$.

- if $\varphi(v) \neq 0$ then the boundary 0 is not reached by $\varphi\left(\lfloor\rfloor_{n}^{-1}(v)\right)$ and it follows that $v$ does not belong to $\left\lfloor V e c t\left\{v^{i} / 1 \leq i \leq k\right\}\right\rfloor_{n}$.

As conclusion, it proves that we just have to compute $\varphi(v)$. If it is null then $v$ belongs to $\left\lfloor V e c t\left\{v^{i} / 1 \leq i \leq k\right\}\right\rfloor_{n}$ and otherwise $v$ belongs to $\left\lfloor V e c t\left\{v^{i} / 1 \leq i \leq\right.\right.$ $k\}\rfloor_{n}$ if and only if $\left.\varphi(v) \in\right]-\varphi(b) ;-\varphi(a)[$. The complexity of this computation is very low. It is the sum of $O\left(n^{2}\right)$ for the computation of $\varphi$ with $O\left(2^{n}\right)$ for the computation of $a$ and $b$, and this global time has nothing to do with the complexity of the investigation of linear inequalities.

\section{Geometrical Case}

We are still interested in the question: does a given vector $v \in \mathbb{Z}^{n}$ belong to the image of $V e c t\left\{v^{i} / 1 \leq i \leq k\right\}$ by the map $\lfloor\cdot\rfloor_{n}$ ?

We suppose now that the constant vector $(1)_{1 \leq j \leq n}$ belongs to $\operatorname{Vect}\left\{v^{i} / 1 \leq\right.$ $i \leq k\}$. In order to simplify the notations and the computations, we are even going to assume that the vectors $v^{1}, v^{2}, \ldots, v^{k}$ are free and that $v^{k}=(1)_{1 \leq j \leq n}$.

\subsection{A Geometrical Equivalent Problem}

Before explaining the consequences of the previous hypothesis, let us see what happens for the question $v \in V e c t\left\{v^{i} / 1 \leq i \leq k\right\}$ ? (for the moment, we forget the integer part $\left.\lfloor\cdot\rfloor_{n}\right)$. The system $\sum_{i=1}^{k} a_{i} v^{i}=v$ can be written $\sum_{i=1}^{k-1} a_{i} v_{j}^{i}-v_{j}=$ $-a_{k}$ for $1 \leq j \leq n$ and it follows that if there exists a solution, the $n$ vectors $\left(v_{j}^{1}, v_{j}^{2}, \ldots ., v_{j}^{k-1}, v_{j}\right)$ containing the $j$ coordinates of $v^{1}, v^{2}, \ldots . v^{k-1}$ and $v$ all belong to an affine hyperplane of $\mathbb{R}^{k}$. Conversely, if the $n$ vectors of coordinates $\left(v_{j}^{1}, v_{j}^{2}, \ldots ., v_{j}^{k-1}, v_{j}\right)$ belong to an affine hyperplane, there exist real coefficients $a_{1}, a_{2}, \ldots, a_{k}$ and $a$ such that $\sum_{i=1}^{k-1} a_{i} v^{i}+a v=-a_{k}(1)_{1 \leq j \leq n}$. The familly $v^{1}, v^{2}, \ldots ., v^{k}=(1)_{1 \leq j \leq n}$ was supposed free and it follows that $a$ can 
not be null (excepting the particular case where $v=0$ ). It proves that $v$ can be written $\sum_{i=1}^{k} a_{i} v^{i}$ if and only if there exists an affine hyperplane of $\mathbb{R}^{k}$ containing the vectors of $j$ coordinates $\left(v_{j}^{1}, \ldots, v_{j}^{k-1}, v_{j}\right)$. It means that the linear question " $v \in \operatorname{Vect}\left\{v^{i} / 1 \leq i \leq k\right\}$ ?" has been transformed by using duallity into a problem of affin geometry. We can do the same with the question " $v \in\left\lfloor V \operatorname{ect}\left\{v^{i} / 1 \leq i \leq k\right\}\right\rfloor_{n}$ ?" with the difference that the part taken by the affine hyperplanes is played by the subsets of $\mathbb{R}^{n}$ characterized by double inequations of the form $h \leq \sum_{i=1}^{n} \alpha_{i} x_{i}<h+\left|\alpha_{n}\right|$. In order to lighten the notations, these sets are simply called the slices of $\mathbb{R}^{n}$ :

Definition 3.1 - - A slice of $\mathbb{R}^{\mathrm{n}}$ is a subset of $\mathbb{R}^{\mathrm{n}}$ which can be characterized by a double inequation of form $\mathrm{h} \leq \sum_{\mathrm{i}=1}^{\mathrm{n}} \alpha_{\mathrm{i} \mathrm{x}_{\mathrm{i}}}<\mathrm{h}+\left|\alpha_{\mathrm{n}}\right|$ where $\mathrm{h}, \alpha_{1}, \alpha_{2}, \ldots . \alpha_{\mathrm{n}}$ are real numbers and where $\alpha_{\mathrm{n}}$ is not null.

We can already notice that the traces of the slices under $\mathbb{Z}^{n}$ are digital hyperplanes [R91]. The affine transformation of the problem in which we are interested provides the following proposition.

Proposition 3.1 - - Let $\mathrm{v}^{1}, \mathrm{v}^{2}, \ldots, \mathrm{v}^{\mathrm{k}-1}, \mathrm{v}^{\mathrm{k}}=(1)_{1 \leq \mathrm{j} \leq \mathrm{n}}$ be a free familly of vectors of $\mathbb{R}^{\mathrm{n}}$ and let $\mathrm{v}$ be an element of $\mathbb{Z}^{\mathrm{n}}$. We have $\mathrm{v} \in\left[\operatorname{Vect}\left\{\mathrm{v}^{\mathrm{i}} / 1 \leq \mathrm{i} \leq \mathrm{k}\right\}\right\rfloor_{\mathrm{n}}$ if and only if the set of the $\mathrm{n}$ vectors $\left(\mathrm{v}_{\mathrm{j}}^{1}, \mathrm{v}_{\mathrm{j}}^{2}, \ldots, \mathrm{v}_{\mathrm{j}}^{\mathrm{k}-1}, \mathrm{v}_{\mathrm{j}}\right)$ of the $j$ coordinates is contained by a slice of $\mathbb{R}^{\mathrm{k}}$.

Proof. If $v \in\left\lfloor V e c t\left\{v^{i} / 1 \leq i \leq k\right\}\right\rfloor_{n}$, it means that the system of $n$ inequalities

$$
v_{j} \leq \sum_{i=1}^{k} a_{i} v_{j}^{i}<v_{j}+1
$$

for $1 \leq j \leq n$, has some solutions. We can rewrite it

$$
-a_{k} \leq \sum_{i=1}^{k-1} a_{i} v_{j}^{i}-v_{j}<-a_{k}+1 .
$$

and it means that the subset of $\mathbb{R}^{k}$ characterized by the double inequation of variable $x=\left(x_{i}\right)_{1 \leq i \leq n} \in \mathbb{R}^{n}$ and of expression

$$
-a_{k} \leq \sum_{i=1}^{k-1} a_{i} x_{i}-x_{k}<-a_{k}+1
$$

contains the $n$ vectors $\left(v_{j}^{1}, v_{j}^{2}, \cdots, v_{j}^{k-1}, v_{j}\right)$ of the $j$ coordinates for $1 \leq j \leq n$. By setting $h=-a_{k}, \alpha_{k}=-1$ and $\alpha_{i}=a_{i}$ for $i$ between 1 and $k-1$, we obtain a first part of the proposition.

Now, let us prove the converse. We suppose that there exists a slice of $\mathbb{R}^{k}$ characterized by a double inequation $h \leq \sum_{i=1}^{k} \alpha_{i} x_{i}<h+\left|\alpha_{k}\right|$ containing the $n$ vectors $\left(v_{j}^{1}, v_{j}^{2}, \cdots, v_{j}^{k-1}, v_{j}\right)$. Before anything, we shall begin by showing that 
$\alpha_{k}$ can be supposed negative. The set of the values taken by the linear form $\sum_{i=1}^{k} \alpha_{i} x_{i}$ for the $n$ vectors $\left(v_{j}^{1}, v_{j}^{2}, \cdots, v_{j}^{k-1}, v_{j}\right)$ is finite. Then there exists a real value $\epsilon>0$ such that the points $\left(v_{j}^{1}, v_{j}^{2}, \cdots, v_{j}^{k-1}, v_{j}\right)$ belong to the set defined by

$$
h \leq \sum_{i=1}^{k} \alpha_{i} x_{i} \leq h+\left|\alpha_{k}\right|-\epsilon .
$$

By multiplying by -1 , it follows that they also belong to the slice defined by

$$
-h-\left|\alpha_{k}\right|+\epsilon \leq \sum_{i=1}^{k}-\alpha_{i} x_{i}<-h+\epsilon .
$$

By setting $h^{\prime}=-h-\left|\alpha_{k}\right|+\epsilon$ and $\alpha_{i}^{\prime}=\alpha_{i}$, we see that $\alpha_{k}$ can be supposed negative and from now, we assume it.

We have for any $j$ between 1 and $n$

$$
h \leq \sum_{i=1}^{k-1} \alpha_{i} v_{j}^{i}+\alpha_{k} v_{j}<h+\left|\alpha_{k}\right| .
$$

It follows that

$$
h /\left|\alpha_{k}\right| \leq \sum_{i=1}^{k-1}\left(\alpha_{i} / \alpha_{k}\right) \cdot v_{j}^{i}-v_{j}<h /\left|\alpha_{k}\right|+1 .
$$

By setting $a_{k}=-h /\left|\alpha_{k}\right|$ and $a_{i}=\alpha_{i} /\left|\alpha_{k}\right|$ for all $i$ between 1 and $k-1$, we have

$$
-a_{k} \leq \sum_{i=1}^{k-1} a_{i} \cdot v_{j}^{i}-v_{j}<-a_{k}+1
$$

and it ends the proof.

The proposition 3.1 transforms the question to know if $v \in\left\lfloor V e c t\left\{v^{i} / 1 \leq i \leq\right.\right.$ $k\}\rfloor_{n}$ into a problem which consists in recognizing the finite parts of slices. In the particular case of finite parts of $\mathbb{Z}^{n}$, this problem has arroused the curiosity of several authors in the previous DGCI ([FST96,DR94]) and it is known as the problem of recognition of digital hyperplanes.

\subsection{Recognition of the Finite Parts of Slices}

The recognition of the finite parts of slices is a problem of convex geometry, which leads to introduce the chords' notion.

Definition 3.2 - Given a part $\mathrm{A} \subset \mathbb{R}^{\mathrm{n}}$, the elements $\mathrm{a}^{\prime}-\mathrm{a}$ where $\mathrm{a} \in \mathrm{A}$ and $\mathrm{a}^{\prime} \in \mathrm{A}$ are called the chords of $\mathrm{A}$. The chords'set of $\mathrm{A}$ is the subset of $\mathbb{R}^{\mathrm{n}}$ denoted chord $(\mathrm{A})$ and defined by

$$
\operatorname{chord}(A)=\left\{a^{\prime}-a / a \in A, a^{\prime} \in A\right\} .
$$


We also introduce the notion of convex hull: the smallest convex part of $\mathbb{R}^{n}$ containing a set $A$ is called the convex hull of $A$ and is denoted $\operatorname{conv}_{R^{n}}(A)$.

These two operators have several properties as the one to commute: we have chord $\circ \operatorname{conv}_{R^{n}}=\operatorname{conv}_{R^{n}} \circ$ chord.

Theorem 3.1 .- There exists a slice of $\mathbb{R}^{\mathrm{n}}$ containing a given finite subset $\mathrm{A} \subset \mathbb{R}^{\mathrm{n}}$ if and only if the point $\mathrm{u}$ of coordinates all null except the last one equal to 1 , does not belong to $\operatorname{conv}_{\mathrm{R}^{\mathrm{n}}}(\operatorname{chord}(\mathrm{A}))$.

It means that we just have to compute if $u=(0,0, \ldots, 0,1)$ belongs to the set $\operatorname{conv}_{R^{n}}(\operatorname{chord}(A))$ in order to know if there exists a slice containing the finite part $A$. Theorem 3.1 can be proved directly but we are going to use an other way based on the elimination of variables according to the method of FoURIERMotzKin.

Proof. According to proposition 3.1, there exists a slice containing the finite set $A=\left\{a^{1}, a^{2}, \ldots, a^{m}\right\}$ if and only if there exist real numbers $h, \alpha_{1}, \alpha_{2}, \ldots, \alpha_{n-1}$ satisfying the system $(\mathrm{S})$ of the $n$ double inequalities

$$
a_{n}^{j} \leq \sum_{i=1}^{n-1} \alpha_{i} a_{i}^{j}-h<a_{n}^{j}+1
$$

for $1 \leq j \leq n$. By eliminating the variable $h$, we obtain the system (S')

$$
1<\sum_{i=1}^{n-1} \alpha_{i}\left(a_{i}^{j}-a_{i}^{j^{\prime}}\right)+\left(a_{n}^{j^{\prime}}-a_{n}^{j}\right)<1
$$

which according to FourIER-Motzkin, is satisfiable if and only if (S) also is. It means that the existence of a slice containing $A$ is equivalent to the existence of a linear form $\varphi\left(x_{i}\right)_{1 \leq i \leq n}=\sum_{i=1}^{n-1} \alpha_{i} x_{i}-x_{n}$ sending the set $\operatorname{chord}(A)$ in ]-1, 1[. As $\operatorname{chord}(A)$ is a symmetric set, it is also equivalent to the existence of a linear form $\varphi\left(x_{i}\right)_{1 \leq i \leq n}=\sum_{i=1}^{n-1} \alpha_{i} x_{i}-x_{n}$ sending the set $\operatorname{chord}(A)$ in $] \varphi(u),+\infty[$. It implies that if there exists a slice containing $A$, then the hyperplane of equation $\varphi(x)=h$ where $\varphi(u)<h<\min (\varphi(\operatorname{chord}(A)))$ stricly separates $\operatorname{chord}(A)$ and the point $u$. The converse is also true: if there exists an hyperplane stricly separating the set $\operatorname{chord}(A)$ and the point $u$, we can write its equation $\varphi\left(x_{i}\right)_{1 \leq i \leq n}=h$ or again $\sum_{i=1}^{n-1} \alpha_{i} x_{i}+\alpha_{n} x_{n}=h$. The coefficient $\alpha_{n}$ can be supposed equal to -1 because it can not be null $(0 \in \operatorname{chord}(A)$ would be on the same hyperplane as $u)$. It follows from the separation and from $\varphi(0)>\varphi(u)$ (the origin belongs to $\operatorname{chord}(A)$ ) that $\varphi(\operatorname{chord}(A)) \subset] \varphi(u),+\infty[$. Then we obtain a new equivalence : there exists a slice containing $A$ if and only if there exists an hyperplane stricly separating $\operatorname{chord}(A)$ and $u$. At this step, the theorem of separation of compact sets [V64] ends the proof.

Theorem 3.1 gives a geometrical characterization of the finite subsets of $\mathbb{Z}^{n}$ contained by slices or in other words, by naive digital hyperplanes and at first 
sight, one could regret that the tools used in the theorem, namely the chords and the convex hull, are continues operators. This critic is howewer not founded because the chords and the discrete convex hull can also be defined in $\mathbb{Z}^{n}$. By denoting them chord and conv $Z^{n}$, the property of commutation is preserved: for any finite set $A \subset \mathbb{Z}^{n}$, we have $\operatorname{chord}\left(\operatorname{conv}_{Z^{n}}\right)(A)=\operatorname{conv}_{Z^{n}}(\operatorname{chord}(A))$ (it is not a trivial result) and it is exactly the trace of the continues set $\operatorname{conv}_{R^{n}}(\operatorname{chord}(A))$ on $\mathbb{Z}^{n}$. It proves the discrete corollary of theorem 3.1 :

Corollary 3.1 .- There exists a digital hyperplane of double inequality

$\mathrm{h} \leq \sum_{\mathrm{i}=1}^{\mathrm{n}} \alpha_{\mathrm{i}} \mathrm{x}_{\mathrm{i}}<\mathrm{h}+\left|\alpha_{\mathrm{n}}\right|$ containing a finite set $\mathrm{A} \subset \mathbb{Z}^{\mathrm{n}}$ if and only if the vector $\mathrm{u}=(0, \cdots, 0,1)$ does not belong to $\operatorname{conv}_{\mathrm{Z}^{\mathrm{n}}}(\operatorname{chord}(\mathrm{A}))$.

In the framework of digital geometry, corollary 3.1 shows that the recognition of finite subsets of slices can be solved by computing the digital convex hull of the chords of the set.

\subsection{Algorithms}

Theorem 3.1 provides a constructive characterization of the finite sets of $\mathbb{R}^{n}$ contained by slices and it provides different geometrical algorithms to recognize them.

The first one is natural. In order to determine if there exists a slice containing the finite part $A \subset \mathbb{R}^{n}$, we begin by computing its chords' set. We obtain a set and we compute its convex hull, by a given method of algorithmic geometry. It only remains to know if the point $u$ belongs to the polytope $\operatorname{conv}_{R^{n}}(\operatorname{chord}(A))$ by determining if there exists a face strictly separating $u$ from the set. If there exists one, then its direction is the direction of a slice containing $A$ and otherwise, there does not exist any solution.

This algorithm can be made incrementaly and several technics improve its effectiveness. The central point of the algorithm is the computation of the convex hull of $\operatorname{chord}(A)$. When we add a point $x$ to the set $A$ (we denote $\left.A^{\prime}=A \cup\{x\}\right)$, the chords' set becomes $\operatorname{chord}\left(A^{\prime}\right)=\operatorname{chord}(A) \cup \operatorname{translation}_{x}(A) \cup$ translation $_{-x}(A)$ and its convex hull can be obtained from $\operatorname{conv}_{R^{n}}(\operatorname{chord}(A))$ and $\operatorname{conv}_{R^{n}}(A)$. We can even notice that if $x \in \operatorname{conv}_{R^{n}}(A)$, then $\operatorname{chord}\left(A^{\prime}\right)=$ $\operatorname{chord}(A)$. Then an incremental algorithm can be obtained by repeating the following process :

We assume that we have computed $\operatorname{conv}_{R^{n}}(A), \operatorname{conv}_{R^{n}}(\operatorname{chord}(A))$ and that $u$ does not belong to this last set. We add the point $x$ to $A$ and obtain $A^{\prime}=A \cup\{x\}$. If $x \in \operatorname{conv}_{R^{n}}(A)$, then $\operatorname{conv}_{R^{n}}\left(A^{\prime}\right)=\operatorname{conv}_{R^{n}}(A)$ and $\operatorname{conv}_{R^{n}}\left(\operatorname{chord}\left(A^{\prime}\right)\right)=$ $\operatorname{conv}_{R^{n}}(\operatorname{chord}(A))$. If $x \notin \operatorname{conv}_{R^{n}}(A)$, then we can use the central step of the incremental Beneath-Beyond method [E87] to compute $\operatorname{conv}_{R^{n}}\left(A^{\prime}\right)$. We also have to compute $\operatorname{conv}_{R^{n}}\left(\operatorname{chord}\left(A^{\prime}\right)\right)$ which is equal to the convex hull of the three convex sets $\operatorname{conv}_{R^{n}}\left(\operatorname{conv}_{R^{n}}(\operatorname{chord}(A)) \cup\right.$ translation $_{-x}\left(\operatorname{conv}_{R^{n}}(A)\right) \cup$ translation $\left._{x}\left(\operatorname{conv}_{R^{n}}(A)\right)\right)$. An efficient method should be a "wrapping" process using the structures of $\operatorname{conv}_{R^{n}}(A)$ and $\operatorname{conv}_{R^{n}}(\operatorname{chord}(A))$ but as we are in dimension $n$, it is not easy. We are then reduced to use the Beneath-Beyond method to obtain $\operatorname{conv}_{R^{n}}\left(\operatorname{chord}\left(A^{\prime}\right)\right)$ without expoiting the convex structure of $\operatorname{conv}_{R^{n}}(A)$. 
This algorithm and its incremental version are natural but they are not optimal. We can wait much better because the structure of the convex polytope $\operatorname{conv}_{R^{n}}(\operatorname{chord}(A))$ (equal to $\left.\operatorname{chord}\left(\operatorname{conv}_{R^{n}}(A)\right)\right)$ seems able to be directly constructed from the structure of the convex polytope $\operatorname{conv}_{R^{n}}(A)$. It is however not yet completly clear.

After this really constructive algorithm, let us present an algorithm coming from the simplex method. There are many different ways of thinking this famous method, and we are going to choose the one which involves the simplexes. Given a finite subset $S$ of $\mathbb{R}^{n}$, the question to determine if a point $y \in \mathbb{R}^{n}$ belongs to the convex hull of $S$ can be solved in searching a simplex whose vertices belong to $S$ and which contains $y$ but instead of going all over the set of all the simplexes, the simplex method is based on the following strategy:

Let us assume that the smallest affine space containing $S$ is $\mathbb{R}^{n}$ because we can also come back to this case, and let us take a $n$-simplex whose vertices $x^{1}, x^{2}, \ldots, x^{n+1}$ belong to $S$. If it contains $y$, then $y$ is in the convex hull of $S$ and it ends the computation, otherwise almost one of the vertices $x^{k}$ in on the opposite side of $y$ towards the face which does not contain it. It provides a linear form $\varphi$ which is negative in $x^{k}$, null in the other vertices and positive in $y$. Then we compute the maximum of $\varphi$ in $S$. If it is null, $y$ does not belong to the convex hull of $S$ and otherwise, the vertices $x^{1}, \ldots, x^{k-1}, x^{\prime k}, x^{k+1}, \ldots, x^{n+1}$ where $x^{\prime k}$ maximizes $\varphi$ in $S$ define our new simplex. We continue in this way until finding a null maximum or a simplex containing $y$. Problems of cycles can sometimes happen but a simple labelling of all the simplexes already met allow to avoid it.

In our problem, we have to determine if the point $u$ belongs to the convex hull of $\operatorname{chord}(A)$ and an easy solution is to use the simplexe method. The points used in the algorithm are, except at the beginning, the one which maximize linear forms and we can notice that given a linear form $\varphi$ and a finite set $A$, a point maximizing $\varphi$ in $\operatorname{chord}(A)$ is the difference $x-x^{\prime}$ between a point $x$ maximizing $\varphi$ in $A$ and $x^{\prime}$ minimizing $\varphi$ in $A$. It means that we do not need to compute the whole set $\operatorname{chord}(A)$ to obtain its maximum by a linear form: with the simplex method, we do not need to compute the chords' set $\operatorname{chord}(A)$. It makes the algorithm very simple and easy to make incremental.

The complexities of the two previous algorithms are not easy to evaluate and we are only going to increase them by rough boundaries. By denoting $a$ the cardinality of the set $A$ and $n$ the dimension, the complexity of the first algorithm is $O\left(a^{2\lfloor(n+1) / 2\rfloor}\right)$ and the fact to make it incremental does not modify it. The complexity of the simplex method is the product between $O\left(a^{2(n+1)}\right)$ and the sum of $O(a)$ with the complexity of the integer computation of the inverse of a matrix of size $n$. This boundary is however excessive because it corresponds to the completly impracticable case where all the $n$-simplexes have to be investigated. We have to compare these values with the complexities of the classical methods solving systems of linear inequalities. The one of the FourIERMotZKIN algorithm is near from $O\left(a^{2^{n}}\right)$ and the one of the method described by EDELSBRUNNER in [E87] is linear in a but still doubling exponential in $n$. 
The complexities of the two geometrical algorithms that we have suggested, are better in $n$ but not in $a$. It remains however probable that in practice, the best of all should be the simplest one, namely the algorithm based on the simplex method.

\section{Conclusion}

The aim of this paper was to bring out the algebra and the geometry hidden in the question "does $v$ belong to the image of a given linear subspace $H$ of $\mathbb{R}^{n}$ by the map $\lfloor.\rfloor_{n}$ ?" but we were not able to do it in the general case. Then we have placed us in particular frameworks and we have obtained the following results:

- If the dimension of $H$ is $n-1$ or $n$, then classical tools of linear algebra allow to conclude.

- If $(1)_{1 \leq i \leq n} \in H$, then the problem is equivalent to the one which consists in determining if there exists a slice containing a given finite part of $\mathbb{R}^{\operatorname{rank}(H)}$ and it can be solved by using the chords and the convex hull. This geometrical translation of the problem can not be generalized to any case. It is specific to this one because the vector $(1)_{1 \leq i \leq n}$ plays a particular role being a kind of step of the function $\lfloor\cdot\rfloor_{n}$. Then the hypothesis is important but in practical, it is not too restrictive. We can as example notice that the problem which consists in computing polynomial approximations belongs to this framework, and then can be solved by the algorithms described at the end.

Generally speaking, the problem of determining if $v$ belongs to $\lfloor H\rfloor_{n}$ has an inexhaustible number of applications and its practical resolution would be a step in the development of what we could call a real digital technology.

\section{References}

DR94. I. Debled-Renesson \& J. P. Reveillès, An incremental algorithm for digital plane recognition. $4^{\text {th }}$ DGCI, Grenoble, France, 1994. 128, 131

D95. I. DeBled-Renesson, Étude et reconnaissance des droites et plans discrets. Thèse de doctorat soutenue à l'Université Louis Pasteur de Strasbourg, 1995. 128

D86. A. DouAdy, Algorithms for computing angles in the Mandelbrot set. Chaotic dynamics and fractals (Atlanta, GA., 1985) pp155-168, Notes Rep. Math. Sci. Engrg., 2, Academic Press, Orlanda, FL, 1986.

E87. H. Edelsbrunner, Algorithms in Combinatorial Geometry. EATCS, Monographs on Theoritical Computer Science, Springer-Verlag, 1987. 127, 133, 134

FST96. J. Françon, J. M. Schramm \& M. TAJine, Recognizing arithmetic straight lines and planes. 6th Conference on Discrete Geometry in Computer Imagery, Lyon, 1996. 128, 131

GA60. D. Gale, The Theory of Linear Economic Models. McGraw-Hill, 1960.

GE99. Y. GERARD, Contribution à la géométrie discrète, thèse de doctorat soutenue ê l'Université d'Auvergne, 1999. 
R91. J. P. REVEILLÈs, Géométrie discrète, calcul en nombre entiers et algorithmique. Thèse de doctorat soutenue à l'Université Louis Pasteur de Strasbourg, 1991. 130

R95. J. P. REveILlès, Combinatorial pieces in digital lines and planes. Vision Geometry IV, vol 2573 SPIE 1995. 128

S88. C. L. Siegel, Lectures on the Geometry of Numbers. Springer-Verlag, 1988. 127

V64. F. VAlentine, Convex sets. McGraw-Hill, series in Higher Mathematics, 1964. 132 